\title{
An Image Reconstruction Method for the X-ray Telescope System with an Angular Resolution Booster
}

\author{
Mikio MorII ${ }^{1}$, Shiro IKedA, ${ }^{1}$ and Yoshitomo MAEdA ${ }^{2}$ \\ ${ }^{1}$ Research Center for Statistical Machine Learning, The Institute of Statistical Mathematics, \\ 10-3 Midori-cho, Tachikawa, Tokyo 190-8562, Japan \\ ${ }^{2}$ Institute of Space and Astronautical Science, Japan Aerospace Exploration Agency, 3-1-1 \\ Yoshinodai, Chuo-ku, Sagamihara, Kanagawa 252-5210, Japan \\ *E-mail: morii@ism.ac.jp \\ Received 2018 October 3; Accepted 2018 November 21
}

\begin{abstract}
We propose an image reconstruction method for an X-ray telescope system with an angular resolution booster proposed by Maeda et al. (2018). The system consists of double multi-grid masks in front of an X-ray mirror and an off-focused two-dimensional imager. Because the obtained image is off-focused, additional image reconstruction process is assumed to be included. Our image reconstruction method is an extension of the traditional Richardson-Lucy algorithm with two regularization terms, one for sparseness and the other for smoothness. Such a combination is desirable for astronomical imaging because astronomical objects have variety in shape from point sources, diffuse sources to mixtures of them. The performance of the system is demonstrated with simulated data for point sources and diffused X-ray sources such as Cas A and Crab Nebula. The image resolution is improved from a few arcmin of focused image without the booster to a few arcsec with the booster. Through the demonstration, the angular resolution booster with the image reconstruction method is shown to be feasible.
\end{abstract}

Key words: techniques: image processing, techniques: high angular resolution, methods: statistical, 


\section{Introduction}

Among X-ray telescopes, Chandra X-ray satellite (Weisskopf et al. 2000) attains the best angular resolution $(0.5$ arcsec). However, the effective area of the focusing mirror is not large enough to collect photons for statistical study of time variation and energy spectrum. X-ray telescopes like Suzaku (Mitsuda et al. 2007) and Hitomi (Takahashi et al. 2016) were designed to have moderately high angular resolution of a few arcmin and large effective area. If the angular resolution of Suzaku/Hitomi telescopes had been improved to a few arcsec preserving the effective area, different scientific topics would be revealed. Two examples are shown below.

The first example is the lensed quasar. Chartas et al. (2017) and references therein monitored several lensed quasars in multi-wavelength including the Chandra X-ray measurements. They found red-shifted and blue-shifted $\mathrm{Fe} \mathrm{K} \alpha$ lines in the spectra of a lensed images. They interpreted the shift of the $\mathrm{Fe} \mathrm{K} \alpha$ line as resulting from gravitational and special relativistic Doppler effects. Since the image separation of lensed quasars is generally larger than a few tenths of arcsec, often down to a few arcsecs, the larger effective area with an angular resolution of arcsecs becomes a unique probe of lensed quasars. The other example is the study of supernova remnants. Uchiyama \& Aharonian (2008); Sato et al. (2018) revealed the mechanism of particle acceleration in supernova remnants by observing time variation of the shell in arcsec resolution for a bright supernova remnant Cas A (Hughes et al. 2000) with Chandra. More of such studies are desired by telescopes with larger effective area than Chandra.

Recently, our companion paper (Maeda et al. 2018) has proposed a simple angular resolution booster for an X-ray telescope like Hitomi, which can improve the angular resolution by order of 1 - 2 possibly with low-cost. The system consists of a set of multi-grid masks in front of the X-ray mirror and two-dimensional imager at a position slightly off-focused from the focal plane. The idea is to utilize the shadow pattern shed on the imager through the masks which contains the information of angular resolution higher than that obtained only with the mirror. Since the imager's position is off-focused, the system needs to reconstruct the sky image from the observed pattern.

For the image reconstruction, the Richardson-Lucy algorithm (Richardson 1972; Lucy 1974) is well-known as a method to obtain the sky image from the data following a Poisson 
statistics. Ikeda et al. (2014) modified the method with a regularizer of sparseness. In this paper, we show a new image reconstruction method by extending these methods with two regularization terms, where one is for sparseness and the other is for smoothness.

Starck, Pantin \& Murtagh (2002) and Puetter, Gosnell \& Yahil (2005) has reviewed image deconvolution methods using Fourier or Wavelet basis for improvement of image resolution, which are different approach from our work.

\section{Mathematical formulation of the problem}

In this article, the image is an $M=m \times n$ pixel rectangular region. This corresponds to a rectangular area on the tangential plane of the celestial sphere. Each pixel is indexed with $u=(i, j)(i=1, \cdots, m ; j=1, \cdots, n)$. The astronomical image is expressed by the distribution of intensity of photons per unit dimension (for example; per unit sky area per unit time, $\mathrm{deg}^{-2}$ $\mathrm{sec}^{-1}$, or per unit sky area per unit time per unit energy range, $\mathrm{deg}^{-2} \mathrm{sec}^{-1} \mathrm{erg}^{-1}$, etc). Here, we express astronomical image $I(u)$ at each pixel $u$ as a non-negative real value. On the other hand, pixels of off-focus plane detector is indexed with $v(v=1, \cdots, V)$. The number of photons in an exposure (an observation) of the telescope detected at a pixel $v$ is $Y(v)$, which is a nonnegative integer value. It follows a Poisson distribution: $Y(v) \sim \operatorname{Poisson}\left(\sum_{u} t(v, u) I(u)\right)$, where $t(v, u)$ is the response of the detector (including the telescope and imager responses) from a pixel $u$ with a unit intensity. We assume each element of the response matrix is a non-negative value and normalized by $\sum_{v} t(v, u)=1$. The normalized image $\rho(u)$ is expressed by $\rho(u)=\frac{I(u)}{S}$, where $S=\sum_{u} I(u)$.

The joint distribution of $Y_{v}$ is described as a product of two probability functions. One is the distribution of $\sum_{v} Y_{v}$, which follows the Poisson distribution Poisson $\left(\sum_{v} Y_{v} ; S\right)$ and the other is that of $Y_{v}$, which follows the multinomial distribution Multinomial $\left(\left\{Y_{v}\right\} ;\left\{\sum_{u} t(v, u) \rho_{u}\right\}\right)$.

$$
\begin{aligned}
& P(Y \mid I)=\prod_{v} \text { Poisson }\left(Y_{v} ; \sum_{u} t(v, u) I(u)\right) \\
= & \frac{e^{-S} S^{\left(\sum_{v} Y_{v}\right)}}{\left(\sum_{v} Y_{v}\right) !} \times \frac{\left(\sum_{v} Y_{v}\right) !}{\prod_{v} Y_{v} !} \prod_{v}\left[\sum_{u} t(v, u) \rho_{u}\right]^{Y_{v}} \\
= & \text { Poisson }\left(\sum_{v} Y_{v} ; S\right) \times \text { Multinomial }\left(\left\{Y_{v}\right\} ;\left\{\sum_{u} t(v, u) \rho_{u}\right\}\right) .
\end{aligned}
$$

The above equation shows $S$ and $\rho$ can be estimated separately. A natural estimate of $S$ is the maximum likelihood estimator, which is easily obtained as $S: S^{*}=\sum_{v} Y(v)$. So, from now on, we consider only the likelihood function of $\rho$. By taking the logarithm and neglecting the terms independent of $\rho$, the likelihood function for $\rho$ becomes $L_{\rho}(\rho)=\sum_{v} Y(v) \log \left[\sum_{u} t(v, u) \rho(u)\right]$. 
It is known that the maximum likelihood of $L_{\rho}(\rho)$ is calculated by the Richardson-Lucy algorithm (Richardson 1972; Lucy 1974). However, when the number of the photons is small, the estimated image becomes unstable. This is because the information required to reconstruct the $m \times n$ image is much larger compared to the limited photon counts.

In order to overcome the problem, we propose a method based on sparse modeling. Sparse modeling is a new signal processing method. Assuming a sparseness of the image in some domain, the amount of information to be estimated is reduced. In this case, we assume the image $\rho$ is sparse (many pixels are zeros) and smooth. Under this assumption, the image would be reconstructed from limited photons. These two types of assumptions is suitable for astronomical imaging, because astronomical objects have variety in shape from point sources, diffuse sources (e.g. supernova remnants, clusters of galaxies, and pulsar wind nebula) to mixtures of them (e.g. point sources in Galactic planes).

Common implementation of the sparse modeling is to use additional regularization terms for the optimization. In our case, we employ a term to encourage the sparseness and another term for smoothness. Widely used regularization term for sparseness is the $\ell_{1}$ norm, but it does not work in this case since $\|\rho(u)\|_{1}=\sum_{u} \rho(u)=1$ from its definition. Thus, we follow the idea in Ikeda et al. (2014), which uses the following term

$$
(1-\beta) \sum_{u} \log \rho(u), \quad 0<\beta .
$$

Here, the parameter $\beta(>0)$ controls the sparseness. Smaller $\beta$ produces sparser image, and the term disappear when $\beta=1$. We call this the Dirichlet term, since it is related to the Dirichlet distribution.

For the regularization of smoothness, we add the following regularization term in the log-likelihood, which works to decrease the difference of pixel values between adjoining pixels:

$$
\begin{aligned}
V(\rho)= & \sum_{i=1}^{m-1} \sum_{j=1}^{n-1}\left[\left(\rho_{i, j}-\rho_{i+1, j}\right)^{2}+\left(\rho_{i, j}-\rho_{i, j+1}\right)^{2}\right] \\
& +\sum_{i=1}^{m-1}\left(\rho_{i, n}-\rho_{i+1, n}\right)^{2}+\sum_{j=1}^{n-1}\left(\rho_{m, j}-\rho_{m, j+1}\right)^{2} .
\end{aligned}
$$

Finally, the cost function of $\rho$ to be minimized becomes

$$
L(\rho)=-L_{\rho}(\rho)+(1-\beta) \sum_{u} \log \rho(u)+\mu V(\rho),
$$

where the hyper parameters $\beta$ and $\mu$ control the degree of sparseness and smoothness, respectively. We optimize $\rho$ in the valid region for sky images: $C=\left\{\rho \in R^{M} \mid \rho_{u} \geq 0, \sum_{u} \rho_{u}=1\right\}$.

The optimization problem of maximizing $L_{\rho}(\rho)$ is known to be solved by the RichardsonLucy algorithm (Richardson 1972; Lucy 1974), which is indeed the EM algorithm for the distri- 
bution obeying Poisson statistics (Dempster, Laird \& Rubin 1977). The problem of maximizing $L_{\rho}(\rho)-(1-\beta) \sum_{u} \log \rho(u)$ is also solved with the EM algorithm as shown in Ikeda et al. (2014). The current problem cannot be solved only with the EM algorithm, then we combined the EM algorithm and the proximal gradient method in order to solve it (e.g. Beck \& Teboulle (2009)).

\section{Algorithm for the reconstruction}

At first, we apply the EM algorithm for the optimization of the cost function, then the $r$-th step in the EM M-step becomes

$$
\rho^{(r+1)}=\underset{\rho \in C}{\arg \min }\left[L_{\mathrm{sub}} \equiv-\sum_{u} m_{u}^{(r)} \log \rho_{u}+(1-\beta) \sum_{u} \log \rho_{u}+\mu V(\rho)\right],
$$

where

$$
m_{u}^{(r)}=\sum_{v} Y(v) \frac{t(v, u) \rho_{u}^{(r)}}{\sum_{u} t(v, u) \rho_{u}^{(r)}}
$$

$(r=1,2, \cdots)$. To solve the sub-problem, we use the proximal gradient method as shown in Beck $\&$ Teboulle (2009). Here, we divide $L_{\text {sub }}$ into two terms $L_{\text {sub }}=g(\rho)+f(\rho)$, where $f(\rho)=\mu V(\rho)$ and $g(\rho)=-\sum_{u}\left[m_{u}^{(r)}-(1-\beta)\right] \log \rho_{u}$. We follow the algorithm of "ISTA with backtracking," then the updating rule of the proximal gradient method in the $k$-th step becomes

$$
\rho^{(k+1)}=\underset{\rho \in C}{\arg \min } \sum_{u}\left[\frac{L_{k}}{2}\left(\rho_{u}-\sigma_{u}^{(k)}\right)^{2}-\left[m_{u}^{(r)}-(1-\beta)\right] \log \rho_{u}\right],
$$

where

$$
\sigma_{u}^{(k)}=\rho_{u}^{(k)}-\frac{\mu}{L_{k}} \frac{\partial V\left(\rho^{(k)}\right)}{\partial \rho_{u}}
$$

$(k=1,2, \cdots)$. Here, the Lipschitz constant $L_{k}$ is searched for in each $k$-step. This minimization problem can be solved in a closed form by introducing a Lagrange multiplier. The details are shown in Appendix. Then, we can solve this minimization problem for a fixed $(\beta, \mu)$. Since the reconstructed image depends on the hyper parameters $(\beta, \mu)$, we determine the best parameters by the cross-validation.

\section{Results}

We test the performance of our proposed reconstruction method by using simulated observed images. To make the simulated images and perform the image reconstruction, we use the response of the detector $t(v, u)$. It is made by a ray-tracing code "xrtraytrace" (Yaqoob et al. 2018). The optics parameter of an Hitomi SXT-like light-weight telescope (Okajima et al. 2016 ) is adopted in the ray-tracing. The angular resolution we set for this ray-tracing is about 

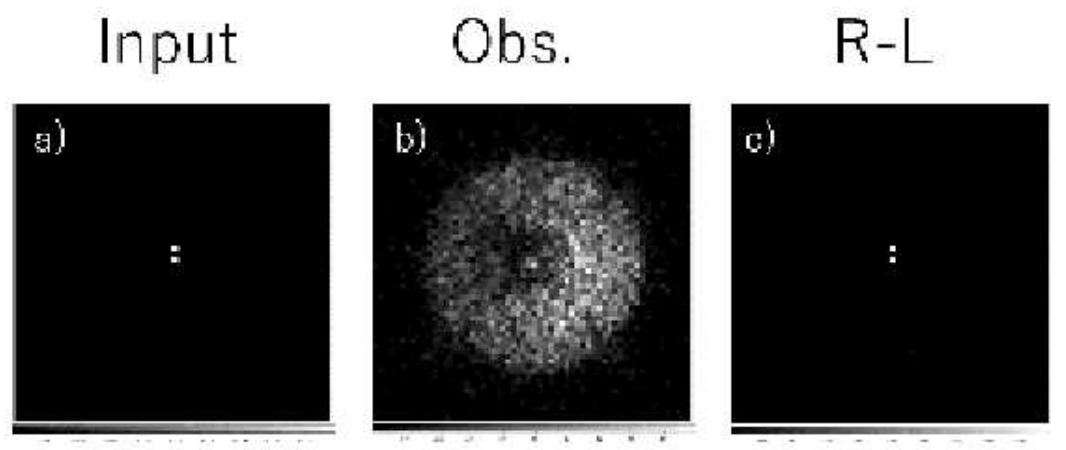

\section{This work}
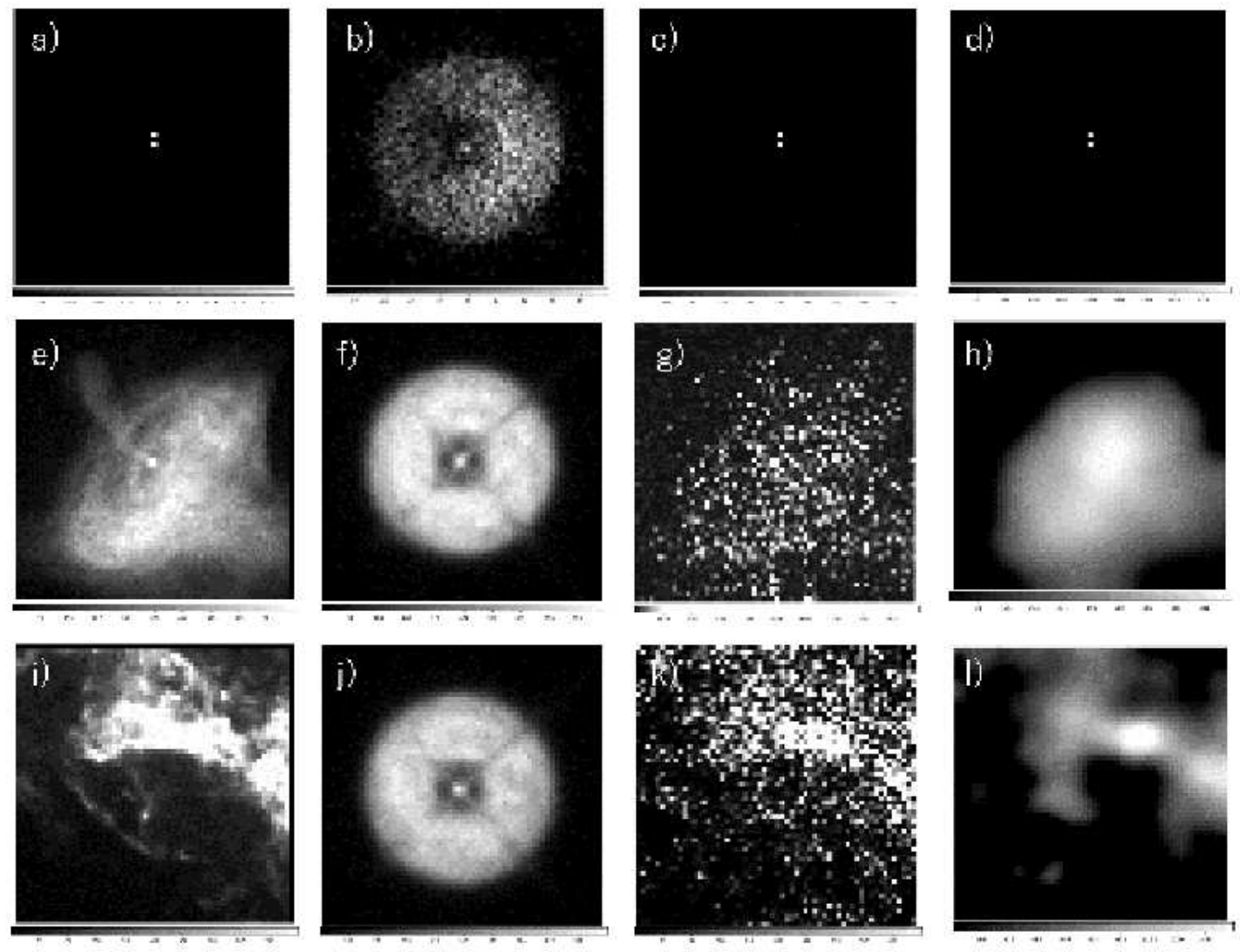

Fig. 1. The image reconstruction for double stars (top), Crab nebula (middle) and Cas A (bottom). The input images are shown in the first column. The panel (a) is double stars with a separation of 4 arcsec. The panels (e, i) are images of Crab nebula and Cas A obtained with Chandra X-ray satellite, respectively. The second columns are simulated observed images on the off-focus imager. The number of photons from the two stars is $5 \times 10^{3}$ photons each (b), and the total number of photons of Crab nebula and Cas A are $10^{6}(\mathrm{f}, \mathrm{j})$. The reconstructed images by the Richardson-Lucy method and our proposed method are shown in the third and fourth columns, respectively. The images $(\mathrm{d}, \mathrm{h}, \mathrm{I})$ are the results with the best hyper parameters, $\left(\beta, \log _{10} \mu\right)=(0.6,1.0),(0.6,8.0)$ and $(0.9,7.0)$, respectively. The pixel scale of the input and reconstructed images are 2 arcsec per one pixel. The images above and elsewhere are made in the look down geometry. 


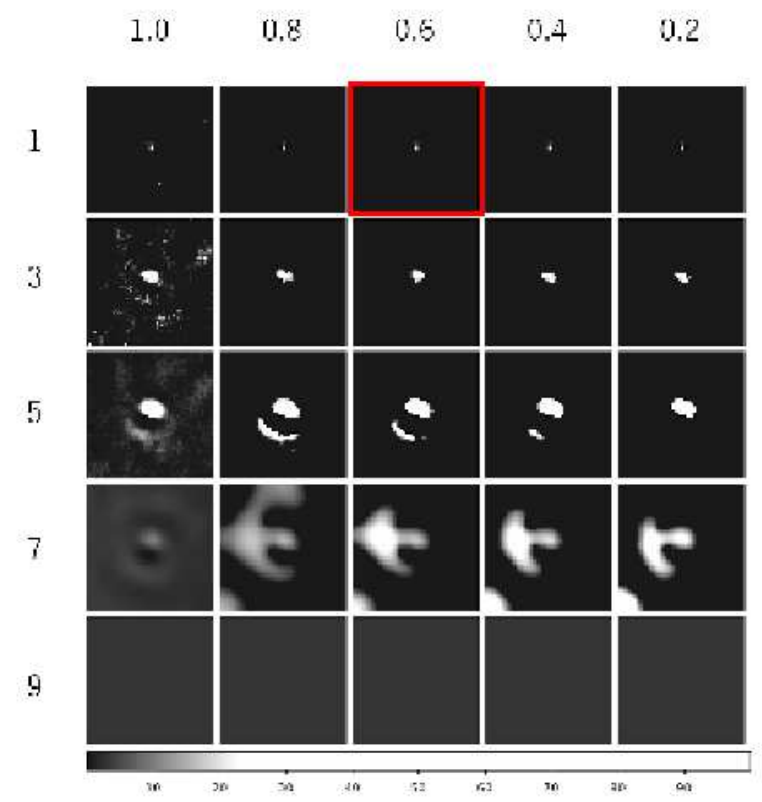

Fig. 2. The reconstructed double stars image by changing hyper parameters $\left(\beta, \log _{10} \mu\right)$. The sparsity and smoothness increase from the left to the right and from the top to the bottom, by setting $\beta$ and $\log _{10} \mu$ as shown above and left of the figure, respectively. The best image determined by the cross-validation is marked with a red frame.

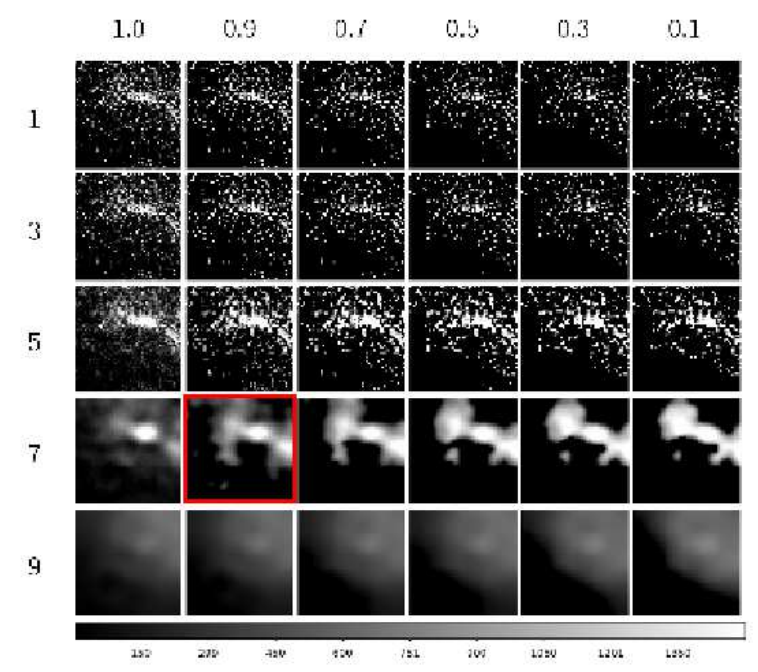

Fig. 3. The similar figure with Figure 2 for Cas A. 
1 arcmin. The off-focus images through the double masks are calculated by offsets with a 2 arcsec pitch. See Maeda et al. (2018) for details.

We simulate images of double point sources with a close separation of 4 arcsec and diffuse sources such as Cas A and Crab Nebula. The input images are shown in Figure 1 (a, e, i) and the simulated observed images on the off-focus imager are the panels (b, f, j), respectively. Both image sizes of the off-focus imager and the reconstructed sky image are $60 \times 60$ pixels. We implemented the algorithm with $\mathrm{C}++$, utilizing BLAS library.

Figures 2 and 3 show the reconstructed images of the double point sources and Cas A along with the hyper parameters varied. The best reconstructed image with the best hyper parameters is determined by the ten-fold cross-validation and shown as the marked panel in these figures. Here, we search the parameter among 121 pairs of $(\beta, \mu): \beta$ is varied from 0.1 to 1.1 by a step of 0.1 , and $\log _{10} \mu$ is varied from 0 to 10 by a step of 1 . The right two columns of Figure 1 presents the reconstructed images for the double stars, Crab Nebula and Cas A obtained by the Richardson-Lucy method and our proposed method.

To estimate the angular resolution of our method, we evaluate the successful separation rate for two close point sources with an angular distance of 4 arcsec as shown in Figure 1 (a) for 100 simulations. In the cases of total photons with $10^{4}, 10^{3}$ and $10^{2}$, the success rates are $100 \%$ (100 out of 100 trials), $99 \%$ (99/100) and 12\% (12/100), respectively.

The processing speed is measured by using a budget computer equipped with Intel Corei7 with $3.20 \mathrm{GHz}$. The image with $60 \times 60$ pixels are reconstructed by $70 \mathrm{sec}$ on average for each hyper-parameter.

\section{Discussion and conclusion}

By the reconstruction of double stars with close separation (Figure 1, d), we demonstrated that the booster system with our image reconstruction method can achieve superb resolution of 4 arcsec, if photon counts from a point source is more than about $5 \times 10^{2}$.

Although the reconstructed images vary with the parameters $(\beta, \mu)$ as shown in Figures 2 and 3 , the best parameters can be determined by the cross-validation, and the best image looks plausible. For point sources, small smoothness parameter is selected by the cross-validation (Figure 2), while for diffuse objects, large smoothness parameters are selected. As shown in Figure 1, the images obtained by the Richardson-Lucy method are noisy, while the images obtained by our method are less noisy and look similar to the input images.

As shown in the second paragraph of Section 2, the total intensity of the X-ray image 
and the pattern of image are estimated separately. Then, the total intensity is simply estimated by $S^{*}$ and one sigma error is by the square root of $S^{*}$. The reconstructed pattern of images depend on the balance between photon statistics and complexity of input images. Roughly speaking, complex images require larger photon statistics than images containing sparse point sources do. Quantitative estimation of necessary number of photons for the reconstruction will be a future work. In this paper, the energy dependency of an X-ray image is not taken into consideration. The reconstruction of image and energy spectrum at once would be the next challenge, e.g. by introducing a regularization of smoothness for energy channel.

In this paper, we have successfully demonstrated that 1-2 order of magnitude improvement of angular resolution can be realized by using angular resolution booster combined with the image reconstruction. This system needs only a simple thus possibly low-cost modification for the current well-established telescopes. Thus, our method will progress X-ray astronomy in near future.

\section{Acknowledgments}

This research is supported by CREST, the Japan Science and Technology Agency (JST), JPMJCR1414, and in part by JSPS Grants-in-Aid for Scientific Research (KAKENHI) Grant No. 17K05395. YM gratefully acknowledges funding from the Tanaka Kikinzoku Memorial Foundation.

\section{Appendix. Solution of the sub-problem}

An iteration step of optimization in the proximal gradient method (Equation 9) is solved by minimizing the following Lagrangian with a Lagrange multiplier $\tau$.

$$
l(\rho, \tau)=\sum_{u}\left[-\left[m_{u}^{(r)}-(1-\beta)\right] \log \rho(u)+\frac{L_{k}}{2}\left(\rho_{u}-\sigma_{u}^{(k)}\right)^{2}\right]+\tau\left(\sum_{u} \rho_{u}-1\right) .
$$

By fixing $\tau$, it is solved in a closed form:

$$
\rho_{u}(\tau)= \begin{cases}\frac{1}{2}\left[b_{u}^{(k)}(\tau)+\sqrt{D_{u}^{(k)}(\tau)}\right] & \left(m_{u}^{(r)}-(1-\beta)>0\right) \\ b_{u}^{(k)}(\tau) & \left(m_{u}^{(r)}-(1-\beta)=0\right) \\ 0 & \left(m_{u}^{(r)}-(1-\beta)<0\right)\end{cases}
$$

where

$$
\begin{aligned}
D_{u}^{(k)}(\tau) & =b_{u}^{(k)}(\tau)^{2}+4 \frac{m_{u}^{(r)}-(1-\beta)}{L_{k}}, \\
b_{u}^{(k)}(\tau) & =\sigma_{u}^{(k)}-\frac{\tau}{L_{k}} .
\end{aligned}
$$

Here, the $\tau$ must satisfy the condition $s(\tau)=\sum_{u} \rho_{u}(\tau)-1=0$. Since the function $s(\tau)$ 
is convex, the solution can be obtained by the Newton-Raphson method. The updating rule of the $i$-th Newton-Raphson step is

$$
\tau^{(i+1)}=\tau^{(i)}-\frac{s\left(\tau^{(i)}\right)}{s^{\prime}\left(\tau^{(i)}\right)},
$$

where

$$
\begin{aligned}
& s^{\prime}(\tau)=\sum_{u} \rho_{u}^{\prime}(\tau), \\
& \rho_{u}^{\prime}(\tau)= \begin{cases}-\frac{1}{2 L_{k}} \frac{b_{u}^{(k)}(\tau)+\sqrt{D_{u}^{(k)}(\tau)}}{\sqrt{D_{u}^{(k)}(\tau)}} & \left(m_{u}^{(r)}-(1-\beta)>0\right) \\
-\frac{1}{L_{k}} & \left(m_{u}^{(r)}-(1-\beta)=0\right) \\
0 & \left(m_{u}^{(r)}-(1-\beta)<0\right)\end{cases}
\end{aligned}
$$

\section{References}

Beck, A. \& Teboulle, M. 2000, SIAM J. Imaging Sci., 2, 183

Chartas, G., et al. 2017, ApJ, 837, 26

Dempster, A. P., Laird, N. M. \& Rubin, D. B. Journal of the Royal Statistical Society. Series B (Methodological), 39, 1

Hughes, J. P. et al. 2000, ApJL, 528, L109

Ikeda, S., Odaka, H., Uemura, M., Takahashi, T., Watanabe, S., \& Takeda, S. 2014, Nuclear Inst. and Methods in Physics Research, A, 760, 46

Lucy, L. B. 1974, AJ, 79, 745

Maeda, Y., Iizuka, R., Hayashi, T., Ishida, M., Sato, T., Ikeda, S., \& Morii, M. 2018, PASJ, submitted

Mitsuda, K. et al. 2007, PASJ, 59, S1

Okajima, T. et al. 2016, Proc. SPIE, 9905, id.99050Z

Puetter, R. C., Gosnell, T. R., \& Yahil, A. 2005, ARA\&A, 43, 139

Richardson, W. H. 1972, Journal of the Optical Society of America, 62, 55

Sato, T. et al. 2018, ApJ, 853, 46

Starck, J. L., Pantin, E., \& Murtagh, F. 2002, PASP, 114, 1051

Takahashi, T. et al. 2016, Proc. SPIE, 9905, 99050U

Uchiyama, Y. \& Aharonian, F. A. 2008, ApJL, 677, L105

Weisskopf, M. C. et al. 2000, Proc. SPIE, 4012, 2

Yaqoob, T. et al. 2018, in preparation 